ISSN 1997-5902

\title{
Activité des extraits de six variétés de piment (Capsicum) utilisés en Cote d'Ivoire
}

\author{
Koffi A.C. (1,2), Koffi-Nevry R. (1), Kouassi K.C. (3), Loukou Y. G. (2) \\ ${ }^{1}$ Laboratoire de Biotechnologie et de Microbiologie Alimentaire de l'UFR des Sciences et Technologies des Aliments \\ de l'Université NANGUI ABROGOUA, 02 BP 801 Abidjan 02 Côte d'Ivoire \\ 2 Laboratoire Nationale de la Santé publique, 18, BP 2403 Abidjan 18, Côte d'Ivoire \\ ${ }^{3}$ Unité de formation et Recherche Agroforesterie, Unité Pédagogique Biochimie-Microbiologie de l'Université Jean \\ Lorougnon Guédé de Daloa, BP150 Daloa, Côte d'Ivoire. \\ Mail de l'auteur correspondant : kofficaro@yahoo.fr tel : 07-09-66-05 / 21-27-99-36
}

Original submitted in on 21 th August 2014. Published online at www.m.elewa.org on 31st October 2014. http://dx.doi.org/10.4314/jab.v82i1.8

\section{RESUME}

Objectif : Évaluer in vitro les activités antifongique de trois extraits de piments (Capsicum) utilisés en Côte d'Ivoire (extrait aqueux, éthanolique $70 \%$ et acétatique) sur la croissance de Penicillium sp. Fusarium sp., Alternaria sp., Aspergillus flavus et Aspergillus niger.

Méthodologies et résultats : La méthode de diffusion en milieu solide et la méthode de diffusion en milieu liquide ont été utilisés pour les tests antimicrobiens. Les résultats obtenus ont révélé une sensibilité de quatre des cinq microbes testés aux différents extraits. Cependant, quelque soit le microbe, l'extrait éthanolique s'est avéré le plus actif avec des valeurs de Concentration Minimale Inhibitrice (CMI) de $84 \mathrm{mg} / \mathrm{mL}$ et de Concentration Minimale Fongicide (CMF) de $168 \mathrm{mg} / \mathrm{mL}$. Par ailleurs, les extraits ont montré des effets fongicide contre Alternaria sp, Penicillium sp., Fusarium sp., Apergillus flavus.

Conclusion et Application : Selon les résultats obtenus, l'éthanol a été le meilleur solvant dans la concentration des principes actifs du piment. En outre, les activités antifongiques des extraits de piments (Capsicum) mise en évidence dans cette étude pourraient justifier l'utilisation du piment dans le traitement de diverses maladies en milieu traditionnel.

Mots clés : Capsicum, piment, antifongique, extrait aqueux, extrait éthanolique, extrait acétatique

\section{Activity of the extracts of six varieties of pepper (Capsicum) used in Côte d'Ivoire}

Objective: To evaluate the in vitro antifungal activities of three extracts from peppers (Capsicum) used in Côte d'Ivoire (aqueous extract, ethanolic $70 \%$ extract and acétatic extract) on the growth Penicillium $\mathrm{sp}$, Fusarium sp, Alternaria sp, Aspergillus flavus and Aspergillus Niger.

Methodology and results: The method of diffusion in solid and the method of dilution in liquid medium were used for antimicrobial testing. The results showed a antifungal sensitivity of four of the five different samples tested molds. However, whatever the microbe, ethanol extract was found to be most active with values of Minimum Inhibitory Concentration (MIC) of $84 \mathrm{mg} / \mathrm{mL}$ and Minimum Fungicide Concentration (MFC) of $168 \mathrm{mg}$ / $\mathrm{mL}$. In addition, all extracts showed fungicidal effects against Alternaria sp., Penicillium sp., Fusarium sp., Apergillus flavus. 
Conclusion and Application: According to the results, ethanol was the best solvent in the concentration of active ingredients of pepper. In addition, the antifungal activities of extracts from peppers (Capsicum) demonstrated in this study may justify the use of pepper in the treatment of various diseases in traditional medecine.

Keywords: Capsicum, pepper, antifungal, aqueous total extract, ethanolic extract, acétatic extract

\section{INTRODUCTION}

Depuis des milliers d'années, l'humanité a utilisé diverses ressources trouvées dans son environnement pour traiter et soigner toutes sortes de maladies (Lee, 2004). Actuellement, I'Organisation Mondiale de la Santé (OMS) estime qu'environ $80 \%$ des habitants de la planète ont recours à la médecine traditionnelle à base de plantes en tant que soins de santé primaire (OMS, 2003 ; Elujoba et al., 2005). L'utilisation des plantes s'explique par leur accessibilité et la disponibilité de la médecine traditionnelle dans les pays en voies de développement d'une part, ainsi que le cout élevé et la nocivité des effets secondaires causés par les médicaments de synthèse d'autre part (Biyiti et al., 2012). Dans toutes les régions du monde, l'histoire des peuples montre que les plantes ont toujours occupé une place importante en médecine, dans la composition des parfums et dans les préparations culinaires (Bouzouita et al., 2008). La flore a déjà énormément contribué à la découverte de nombreux principes actifs qui ont servi à la préparation de nombreux médicaments et elle y contribue encore (Kporou et al., 2009). En effet, l'industrie pharmaceutique moderne elle-même, continue de s'appuyer sur la diversité des métabolites secondaires végétaux pour trouver de nouvelles molécules aux propriétés biologiquement actives (Kra, 2001). Les plantes médicinales contiennent de grandes variétés de substances chimiques qui possèdent des propriétés thérapeutiques importantes qui peuvent être utilisées dans le traitement de maladies humaines. De nombreux médicaments couteux actuellement utilisés ne sont pas disponibles dans les zones rurales. La médecine traditionnelle ivoirienne utilise une grande variété de plantes pour traiter les troubles gastro-intestinaux tels que la diarrhée (Koffi-Nevry et al., 2012). Dans les zones rurales de la Côte d'Ivoire, les infections sont traitées avec le médicament le plus accessible. Les maladies d'origine alimentaire constitue une préoccupation de santé publique pour les pays développés et en voie de développement (Akinpelu et al., 2008 ). Avec l'évolution de la société, le consommateur est devenu plus soucieux de la sécurité alimentaire, et il demande à être protégé de mieux en mieux contre les risques de contamination. Tandis que les professionnels de l'agro-industrie prennent des mesures de plus en plus strictes pour garantir l'innocuité des aliments, les pouvoirs publics mettent également en place des normes de plus en plus sévères pour prévenir au mieux, les risques sanitaires (El-Koury, 2007). Parmi les éléments thérapeutiques de la médecine, le piment est toujours cité (Dorantes et al., 2000). Les fruits de Capsicum sont très appréciés dans l'alimentation. Le piment est essentiel dans les mets africain. En Côte d'Ivoire, certains mets sont reconnus pour leur forte teneur en piment notamment le Kedjénou (soupe de poulet et le Biokesseu (soupe de poisson) (Kouassi, 2012). Connus aussi comme plante médicinale, les fruits de Capsicum sont employés en médecine traditionnelle pour leurs propriétés antimicrobiennes dues aux métabolites secondaires qu'ils contiennent (Hervet-Hernandez et al., 2010 ; Kouassi et al., 2012). Des études antérieures ont montré que les différentes variétés de piments cultivées en Côte d'Ivoire ont des activités antimicrobiennes sur des souches bactériennes pathogènes (Escherichia coli, Vibrio cholerae, Staphylococcus aureus, Pseudomonas aeruginosa) et sur les souches levuriennes (Candida) (Kouassi et al., 2012). Cependant aucune étude n'a été encore entreprise à notre connaissance sur l'activité des extraits du piment sur des moisissures. Or parmi les microorganismes pathogènes responsables des infections courantes, les champignons occupent une place importante. Le présent travail a pour objectif d'évaluer l'activité antifongique de six variétés de piments consommées en Côte d'Ivoire sur Aspergillus niger, Aspergillus flavus, Penicillium sp., 
Koffi et al. J. Appl. Biosci. 2014. Activité des extraits de six variétés de piment (Capsicum) utilisés en Cote d'Ivoire

Alternaria sp. Et Fusarium sp. Ces champignons pathogènes sont responsables de toxi-infection

\section{MATERIEL ET METHODES}

Matériel végétal: Le matériel végétal utilisé est constitué de 6 variétés de piments commercialisées sur le marché d'Abidjan : Capsicum annuum var. antillais, Capsicum frutescens var. doux, Capsicum frutescens var. soudanais, Capsicum frutescens var. attié, Capsicum frutescens oiseau, Capsicum chinense var. pendulum. Ces variétés de piments ont été collectées sur cinq grands marchés dans le district d'Abidjan: Abobo, alimentaire.

Yopougon, Adjamé, Treichville et Port-Bouët. Les fruits de Capsicum annuum et Capsicum chinense choisi étais frais tandis que les fruits de Capsicum frutescens choisi ont été séché parce qu'utilisé dans cet état. Ces différentes variétés ont étés identifiées au Centre National de Floristique de I'Université Félix HOUPHOUËT- BOIGNY Abidjan.

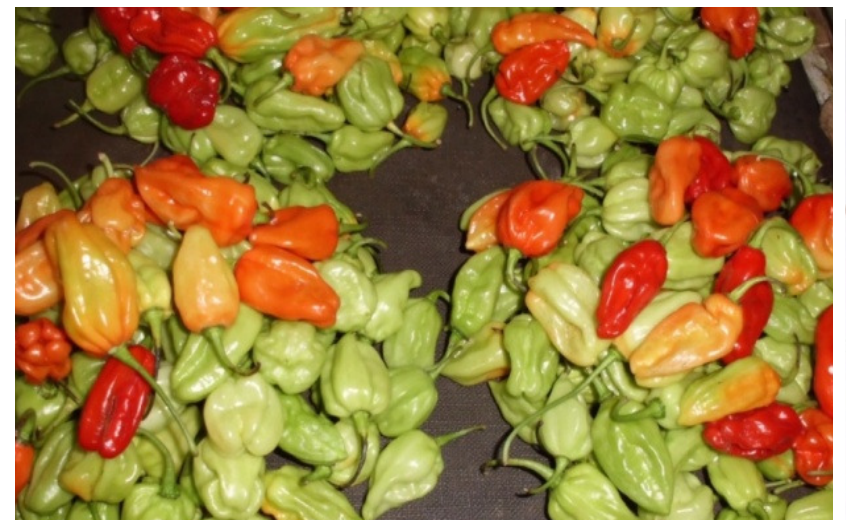

Figure 1 : Capsicum annuum variété antillais

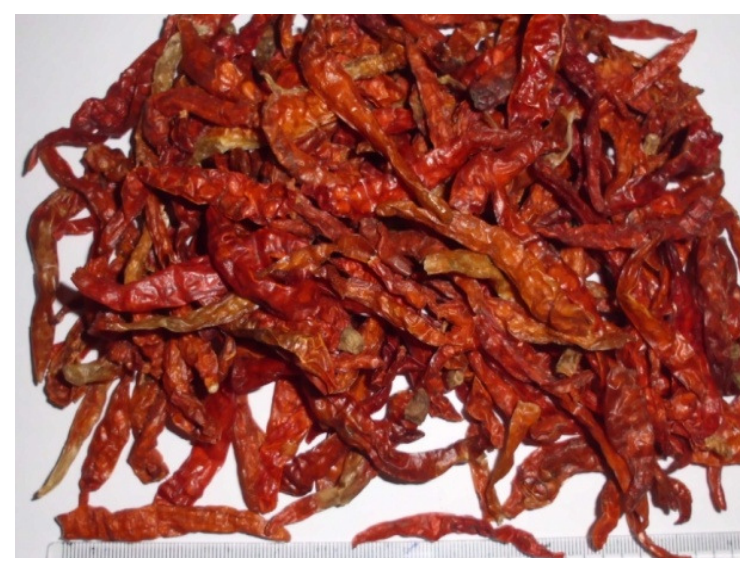

Figure 3 : Capsicum frutescens variété attié

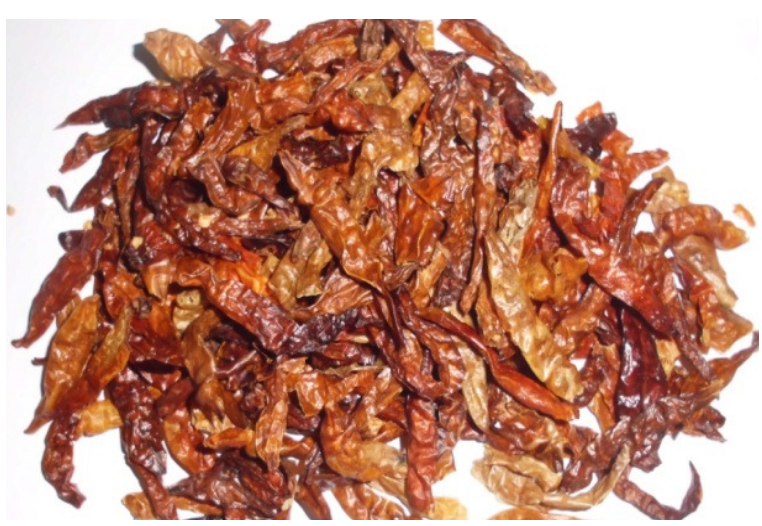

Figure 2 : Capsicum frutenscens variété doux

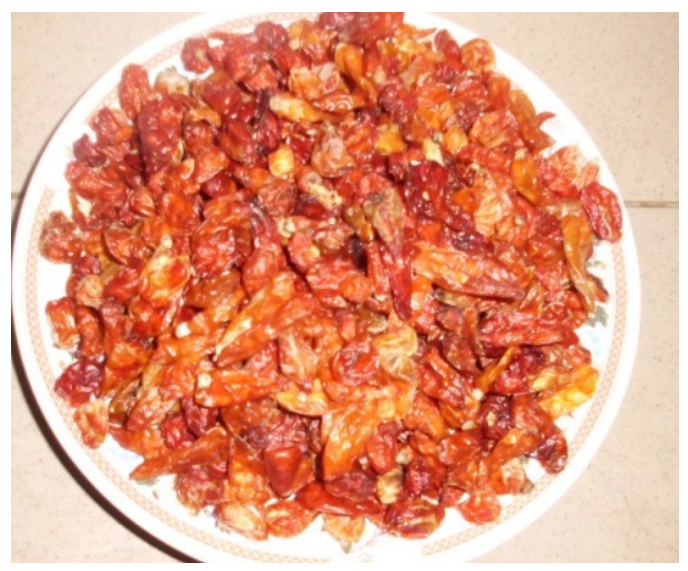

Figure 4 : Capsicum frutescens variété Soudanais 


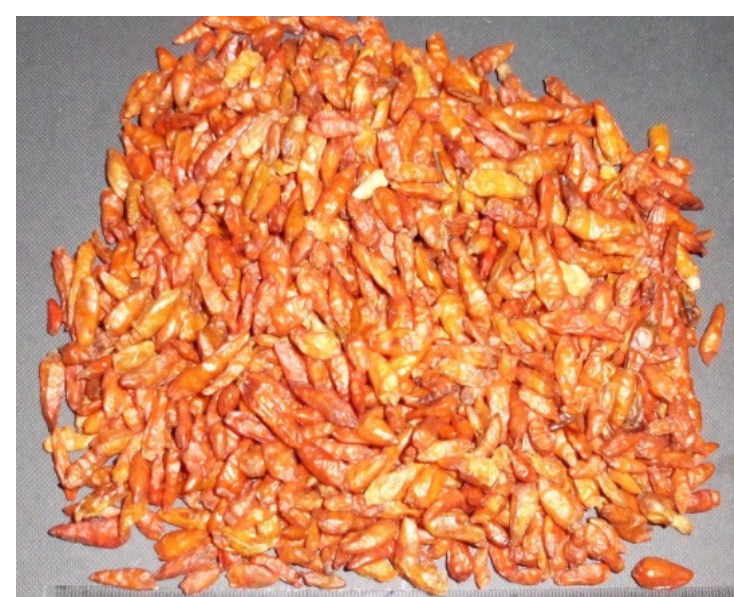

Figure 5 : Capsicum frutescens variété Oiseau

Matériel biologique: II est constitué de 5 souches fongiques : Aspergillus niger, Fusarium sp., isolées des échantillons de cola, Alternaria sp., Aspergillus flavus isolées des sons de blé et Penicillium sp., isolée dans une industrie de la place. Toutes ces souches ont été fournies par l'Institut Pasteur de Côte d'Ivoire. Ces différentes souches fongiques ont été repiquées incubées et conservées dans la gélose de conservation à la température de réfrigération à $+4^{\circ} \mathrm{C}$. Les cultures sont renouvelées tous les 15 jours.

Préparation des extraits végétaux: Les piments sélectionnés ont été triés, lavés et séchés à l'étuve à $55^{\circ}$ $C$ séparément pendant 5 jours pour les fruits de Capsicum frutescens et 8 à 10 jours pour les fruits de Capsicum annuum et Capsicum chinense. Les fruits séchés ont été pulvérisés dans un mixeur électrique (Blender 8010E modèle 38BL40) à $3000 \mathrm{trs} / \mathrm{min}$ ). Le mélange obtenu a été tamisés (mailles environ 1-2 mm de diamètre). Les poudres obtenues ont servis à réaliser les extraits. Les extraits ont été préparés selon la méthode décrite par N'guessan et al., 2007 et Angeh (2006) . Trois solvants (eau distillée stérile, l'éthanol et l'acétate d'éthyle) ont été utilisés. L'extrait aqueux a été obtenu en dissolvant $20 \mathrm{~g}$ de poudre dans $200 \mathrm{~mL}$ d'eau distillée bouillante puis porté à ébullition pendant $15 \mathrm{~min}$. L'homogénat obtenu est filtré sur du papier Whatman №2. Le filtrat obtenu a été transféré dans une fiole et évaporation à $50^{\circ} \mathrm{C}$. Le filtrat séché constitue l'extrait total aqueux (Etaq). L'extrait éthanolique (Eeth) a été obtenu en dissolvant dix gramme $(10 \mathrm{~g})$ de l'extrait aqueux dans $200 \mathrm{~mL}$ d'une solution d'éthanol à 70\% puis homogénéisé pendant 24 heures à l'aide d'un agitateur magnétique. Après filtration sur du papier Whatman $\mathrm{N} 02$, le filtrat recueilli a été évaporé à l'étuve à $50^{\circ} \mathrm{C}$. La poudre obtenue constitue l'extrait éthanolique. Cinq (5) g de

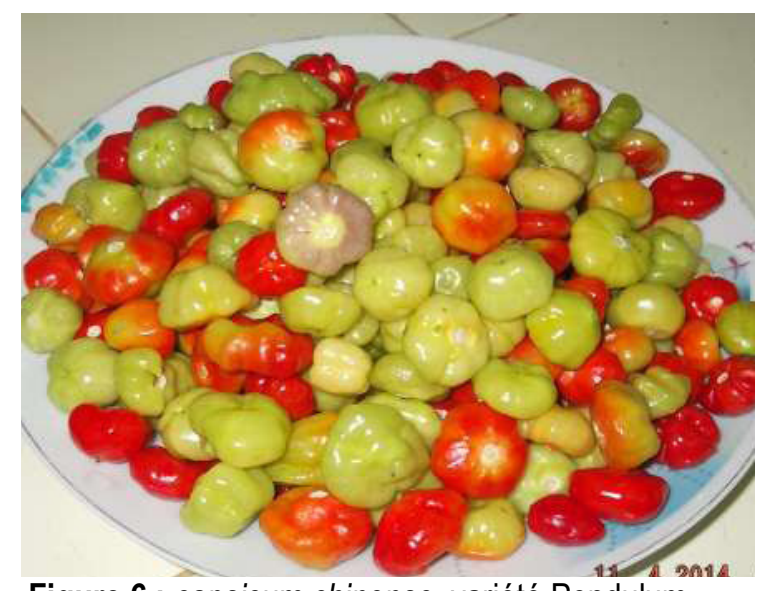

Figure 6 : capsicum chinense variété Pendulum

poudre ont été homogénéisé dans $200 \mathrm{~mL}$ d'une solution composée d'un mélange d'acétate d'éthyle et d'eau distillée pendant 24 heures à l'aide d'un agitateur magnétique. A l'aide d'une ampoule à décanter, les fractions d'eau et l'acétate d'éthyle ont été recueillis et séché à l'étuve à $50^{\circ} \mathrm{C}$. La fraction séchée d'acétate d'éthyle obtenue constitue l'extrait acétatique (Eace). Tous ces extraits ont été testés séparément sur la croissance des différents champignons étudiés.

\section{Activité antifongique}

Préparation de la gélose Sabouraud: L'ensemencement s'est fait sur la gélose de Sabouraud glucosée (BIOMEDIS). Le milieu a été préparé selon les indications du fabricant et coulé dans des boîtes de Pétri. Préparation de la gamme de concentration: La méthode de dilution en milieu solide à été utilisée pour voir la sensibilité des souches face aux extraits à une concentration de $672 \mathrm{mg} / \mathrm{mL}$. A l'issue de cette étude, pour tout extrait sur une souche fongique présentant une zone d'inhibition a été retenue pour la détermination de la $\mathrm{CMI}$ en milieu liquide. Une gamme de concentration de l'extrait a été préparée dans sept tubes à essais à partir d'une solution mère de concentration $672 \mathrm{mg} / \mathrm{mL}$ selon une progression géométrique de raison 2 de manière à obtenir les concentrations de $336 \mathrm{mg} / \mathrm{mL}, 168 \mathrm{mg} / \mathrm{mL}, 84$ $\mathrm{mg} / \mathrm{mL}, 42 \mathrm{mg} / \mathrm{mL}, 21 \mathrm{mg} / \mathrm{mL}$.

Préparation de l'inoculum fongique: Les tests antifongiques ont été réalisés avec de jeunes cultures de moisissures de 24 à 48 heures obtenues sur boîte de Pétri. A partir de ces cultures, 2 à 3 colonies ont été prélevées et mélangées dans $2 \mathrm{~mL}$ d'eau physiologique stérile contenue dans un tube à hémolyse stérile. Une fois bien homogénéisée, la suspension est lue au Densimat (ref. 99535 A version, Biomerieux) en l'ajustant jusqu'à obtenir l'opacité de $0,5 \mathrm{Mc}$ Farland ou à un DO de 
0,08 à 0,1 lue à $625 \mathrm{~nm}$. L'inoculum final de l'espèce a été obtenu en ajoutant $100 \mu \mathrm{l}$ de cette suspension à 10 $\mathrm{mL}$ d'eau physiologique stérile. Cette suspension finale contenant environ $10^{8} \mathrm{UFC} / \mathrm{mL}$ a constitué l'inoculum fongique de dilution $10^{\circ}$ ou l'inoculum pur.

Numérotation de l'inoculum : $L$ 'inoculum fongique a été dilué de 10 en 10 jusqu'à la dilution $10^{-4}$. On obtient quatre dilutions successive de $10^{-1}$ à $10^{-4}$. Ces différentes dilutions ainsi que l'inoculum pur ont été ensemencés à l'aide d'une anse calibrée de $2 \mu \mathrm{L}$ par stries de $5 \mathrm{~cm}$ de long sur gélose Sabouraud puis incubés à $25^{\circ} \mathrm{C}$ pendant $72 \mathrm{~h}$. Cette préparation a constitué la boîte $A$.

Ensemencement: L'inoculum pur préalablement préparé est versé sur la surface de la gélose Sabouraud coulée en boîte de Pétri avec une épaisseur de $4 \mathrm{~mm}$. Après un temps de contact de 5 minutes, le surplus de l'inoculum est retiré avec une micropipette. A l'aide d'un écouvillon stérile, l'excès de l'inoculum est retiré par des pressions sur les bords de la boîte en l'inclinant de $60^{\circ}$. La boîte est séchée à $37^{\circ} \mathrm{C}$ pendant $5 \mathrm{~min}$.

Sensibilité des souches face aux extraits: La sensibilité des souches aux extraits de Capsicum a été réalisée par la technique de diffusion en milieu gélosé. La méthode des trous en l'emporte pièce dans la gélose a été retenue aux dépens de la méthode des disques chargés en raison des limites observées dans cette dernière méthode lors des études préliminaires (non diffusion de l'extrait). Le principe de cette méthode des puits repose sur la diffusion du composé antimicrobien en milieu solide. Dans une boîte de Pétri, à partir d'un point précis, (trou ou puits) la substance antimicrobienne active diffuse avec création d'un gradient de concentration après un temps de contact entre celle-ci et le microorganisme (Suffredini, (2006) ; Vinod et al. (2010) ; Kouassi (2012). La partie réservoir d'une pipette Pasteur stérile est enfoncée dans la gélose jusqu'à toucher le fond de la boîte. La pipette est retournée lentement sur elle-même et retirée en l'inclinant légèrement. Ces puits d'environ $6 \mathrm{~mm}$ de diamètre sont effectués dans la gélose. Un volume de $70 \mu \mathrm{L}$ des différents extraits de Capsicum à tester à la concentration de $672 \mathrm{mg} / \mathrm{mL}$ sont administrés dans chaque puits. Les boîtes de Pétri on été laissée 2 à 3 heures à la chambre froide $\left(5\right.$ à $\left.10^{\circ} \mathrm{C}\right)$ pour permettre la diffusion des extraits dans la gélose. Les boîtes de Pétris ont été incubées à $25^{\circ} \mathrm{C}$ pendant $72 \mathrm{~h}$.

\section{RESULTATS}

Activité antifongique: Les résultats des diamètres d'inhibition des différentes variétés de Capsicum sur les souches fongiques sont présentés dans le tableau 1. Les différents extraits des variétés de piments sont actifs sur
La présence ou non d'une zone d'inhibition (pas de colonies autour du puits) a été observée et mesurée à l'aide d'une règle. Parallèlement, 6 antifongiques (Amphotéricine B, Econasole, Clotrimazole, Miconazole, Nistatine, Ketonazole) usuels ont été utilisés pour le contrôle positif et pour le contrôle négatif, $70 \mu \mathrm{L}$ d'eau distillée stérile et d'éthanol on été utilisé. Des diamètres d'inhibition ont été mesurés pour les souches sensibles. Les CMI et les CMF ont été ensuite déterminées.

Détermination de la CMI et de la CMF : La Concentration Minimale Inhibitrice (CMI) a été déterminée en milieu liquide tandis que la Concentration Minimale Fongicide (CMF) a été déterminé en milieu gélosé. La $\mathrm{CMI}$ est la plus faible concentration de substance pour laquelle il n'y a pas de croissance visible à l'œil nu après un temps d'incubation de $72 \mathrm{~h}$. Sa détermination a été faite par observation du trouble induit par la croissance des microbes étudiés dans chaque tube. La CMF est la plus petite concentration d'extrait qui donne $99,99 \%$ d'inhibition comparativement au tube témoin de contrôle de croissance ou inversement, c'est la concentration qui laisse une survivance de 0,01 \% par rapport au tube témoin de contrôle de croissance. Dans une série de 7 tubes à hémolyse numéroté de $T_{1}$ à $T_{7}$, un tube témoin pour la croissance des microbes et un tube témoin pour le test de stérilité du milieu de culture l'extrait a été introduit $1 \mathrm{~mL}$ de l'inoculum pur. Ensuite, $1 \mathrm{~mL}$ d'extrait végétal selon la gamme de concentration préparée a été ajouté dans chaque tube. La série de tube contient ainsi des concentrations de l'extrait allant de 672 à $21 \mathrm{mg} / \mathrm{mL}$. Ces tubes ont été incubés à $25^{\circ} \mathrm{C}$ pendant 72 heures. L'étude a été reprise trois fois. Ainsi, la CMI a été donc la concentration du premier tube à partir duquel aucun trouble à l'œil nu n'a été observé. Le contenu des tubes dans lesquels aucun trouble n'a été observé a servi à ensemencer la gélose Sabouraud sur des stries de $5 \mathrm{~cm}$ en commençant par le premier tube sans trouble. Après 72 heures d'incubation à $37^{\circ} \mathrm{C}$. La Concentration Minimale Fongicide (CMF) a été déterminé en comparant le nombre de colonie sur les stries (boîte B) à celle de la boîte de numérotation de l'inoculum (boîte A). Ainsi, le premier tube expérimental dont le nombre de microbes présent sur sa strie est inférieur ou égale à celui de la dilution $10^{-4}$ correspondra à la CMB.

quatre des cinq souches testées à savoir : Alternaria $\mathrm{sp}$.Penicillium sp, Fusarium sp et Aspergillus flavus. Toute fois, ces différents extraits n'ont eu aucune action sur $A$. niger. La réceptivité des micro-organismes s'est 
avérée variable selon la souche testée et selon le type d'extrait. Bien que la réponse, n'ai pas été uniforme, tous les extraits de Capsicum ont montré une activité sur ces quatre souches fongiques. Les extraits aqueux et éthanoliques ont été plus actifs sur les souches Alternaria sp., Penicillium sp. et Fusarium sp. avec des diamètres d'inhibitions allant de $12 \mathrm{~mm}$ à $18 \mathrm{~mm}$ pour l'extrait aqueux et de $12 \mathrm{~mm}$ à $19 \mathrm{~mm}$ pour l'extrait éthanolique. Cependant, une faible sensibilité a été observée avec l'extrait éthanolique des variétés attié et doux avec des diamètres d'inhibitions allant de $6 \mathrm{~mm}$ à $9 \mathrm{~mm}$ sur la souche Alternaria. Les extraits de Capsicum chinense variété pendulum ont montré une forte activité antifongique sur les moisissures testées. Les extraits aqueux et éthanoliques de trois variétés (antillais, doux et pendulum) ont présenté des zones d'inhibitions sur Penicillium respectivement de $16 \mathrm{~mm}$ à $18 \mathrm{~mm}$ pour l'extrait aqueux et de $16 \mathrm{~mm}$ à $19 \mathrm{~mm}$ pour l'extrait éthanolique. Les extraits éthanoliques et acétatique ont inhibé la croissance de Fusarium avec des diamètres de $14 \mathrm{~mm}$ à $16 \mathrm{~mm}$ Tout ceci à la concentration de 672 $\mathrm{mg} / \mathrm{mL}$. Cependant aucun des extraits n'à eu d'effet sur la croissance de Aspergillus niger. II constitue donc la souche la plus résistante. La souche la plus sensible est Alternaria L'extrait le plus actif est l'extrait éthanolique, le moins actif est l'extrait acétatique.

Tableau 1 : Diamètre (mm) d'inhibition des différentes variétés de Capsicum.

\begin{tabular}{|c|c|c|c|c|c|c|}
\hline $\begin{array}{l}\text { Variétés } \\
\text { Capsicum }\end{array}$ & Extraits & Alternaria & Penicillium & Fusarium & A.flavus & A.niger \\
\hline $\begin{array}{l}\text { Capsicum annuum } \\
\text { antillais }\end{array}$ & $\begin{array}{c}\text { Aqueux } \\
\text { Ethanolique } \\
\text { Acétatique }\end{array}$ & $\begin{array}{c}17,66 \pm 0,57 \\
18 \pm 2 \\
17,50 \pm 0,57\end{array}$ & $\begin{array}{c}16 \pm 1,73 \\
17,33 \pm 0,57 \\
-\end{array}$ & $\begin{array}{c}- \\
16,33 \pm 0,57 \\
16 \pm 1\end{array}$ & $\begin{array}{l}- \\
- \\
-\end{array}$ & $\begin{array}{l}- \\
- \\
-\end{array}$ \\
\hline $\begin{array}{l}\text { Capsicum frutescens } \\
\text { soudanais }\end{array}$ & $\begin{array}{l}\text { Aqueux } \\
\text { Ethanolique } \\
\text { Acétatique }\end{array}$ & $\begin{array}{c}14,66 \pm 0,57 \\
18 \pm 1 \\
-\end{array}$ & $\begin{array}{l}- \\
- \\
-\end{array}$ & $\stackrel{-}{-}$ & $\begin{array}{l}- \\
- \\
-\end{array}$ & $\begin{array}{l}- \\
- \\
-\end{array}$ \\
\hline $\begin{array}{l}\text { Capsicum frutescens } \\
\text { attié }\end{array}$ & $\begin{array}{l}\text { Aqueux } \\
\text { Ethanolique } \\
\text { Acétatique }\end{array}$ & $\begin{array}{c}12,33 \pm 0,57 \\
6,66 \pm 1,52 \\
-\end{array}$ & $\begin{array}{l}- \\
-\end{array}$ & $\begin{array}{l}- \\
-\end{array}$ & $\begin{array}{l}- \\
-\end{array}$ & $\begin{array}{l}- \\
-\end{array}$ \\
\hline $\begin{array}{l}\text { Capsicum frutescens } \\
\text { doux }\end{array}$ & $\begin{array}{l}\text { Aqueux } \\
\text { Ethanolique } \\
\text { Acétatique }\end{array}$ & $\begin{array}{c}12,33 \pm 0,57 \\
9 \pm 1 \\
-\end{array}$ & $\begin{array}{c}- \\
16,33 \pm 0,57 \\
-\end{array}$ & $\begin{array}{l}- \\
-\end{array}$ & $\begin{array}{c}- \\
- \\
12 \pm 0,57\end{array}$ & $\begin{array}{l}- \\
-\end{array}$ \\
\hline $\begin{array}{l}\text { Capsicum frutescens } \\
\text { oiseau }\end{array}$ & $\begin{array}{l}\text { Aqueux } \\
\text { Ethanolique } \\
\text { Acétatique }\end{array}$ & $\begin{array}{c}15,66 \pm 1,15 \\
12 \pm 1 \\
-\end{array}$ & $\begin{array}{l}- \\
- \\
-\end{array}$ & $\begin{array}{c}- \\
14 \pm 1 \\
-\end{array}$ & $\begin{array}{l}- \\
- \\
-\end{array}$ & $\begin{array}{l}- \\
- \\
-\end{array}$ \\
\hline $\begin{array}{l}\text { Capsicum chinense } \\
\text { pendulum }\end{array}$ & $\begin{array}{l}\text { Aqueux } \\
\text { Ethanolique } \\
\text { Acétatique }\end{array}$ & $\begin{array}{l}18,33 \pm 1,52 \\
19,66 \pm 0,57 \\
17,50 \pm 0,57\end{array}$ & $\begin{array}{c}18,33 \pm 1,52 \\
19 \pm 1 \\
-\end{array}$ & $\begin{array}{c}- \\
15 \pm 1 \\
16 \pm 1\end{array}$ & $\begin{array}{c}- \\
- \\
14 \pm 0,57\end{array}$ & $\begin{array}{l}- \\
-\end{array}$ \\
\hline
\end{tabular}

A.flavus : Aspergillus flavus ,A.niger : Aspergillus niger ; (-) : absences de zones d'inhibition

Les antifongiques usuels utilisés dans cette étude ont montré pour la plupart, des zones d'inhibitions considérables avec des valeurs allant de $12 \mathrm{~mm}$ à $48 \mathrm{~mm}$ de diamètre sur les cinq souches fongiques étudiées (tableau 2). 
Koffi et al. J. Appl. Biosci. 2014. Activité des extraits de six variétés de piment (Capsicum) utilisés en Cote d'Ivoire

Tableau 2 : Diamètre $(\mathrm{mm})$ des zones d'inhibitions des antifongiques usuels

\begin{tabular}{l|c|c|c|c|c}
\hline \multirow{2}{*}{ antifongiques } & \multicolumn{5}{|c}{ Diamètre d'inhibition (mm) } \\
\cline { 2 - 6 } & Alternaria & Penicillium & Fusarium & A.flavus & A.niger \\
\hline CTR 50 & $48(\mathrm{~S})$ & $0(\mathrm{R})$ & $24(\mathrm{~S})$ & $22(\mathrm{~S})$ & $12(\mathrm{~S})$ \\
MCZ 50 & $36(\mathrm{~S})$ & $22(\mathrm{~S})$ & $22,2(\mathrm{~S})$ & $18(\mathrm{~S})$ & $16(\mathrm{~S})$ \\
NY 100 & $17(\mathrm{~S})$ & $15(\mathrm{~S})$ & $12(\mathrm{~S})$ & $12(\mathrm{~S})$ & $13(\mathrm{~S})$ \\
EC 50 & $44(\mathrm{~S})$ & $24(\mathrm{~S})$ & $28(\mathrm{~S})$ & $33(\mathrm{~S})$ & $30(\mathrm{~S})$ \\
AB 100 & $15(\mathrm{~S})$ & $14(\mathrm{~S})$ & $12(\mathrm{~S})$ & $13(\mathrm{~S})$ & $12(\mathrm{~S}$ \\
KET 50 & $56(\mathrm{~S})$ & $28(\mathrm{~S})$ & $40(\mathrm{~S})$ & $26(\mathrm{~S})$ & $4(\mathrm{R})$ \\
\hline
\end{tabular}

CTR 50: Clotrimazole $50 \mu \mathrm{g}$; MCZ 50: Miconazole $50 \mu \mathrm{g}$; NY100 : Nystatine $100 \mathrm{UI}$; EC 50 :Econazole $50 \mu \mathrm{g}$; AB100 :Amphotéricine B100 $\mu \mathrm{g}$; KET 50 : Ketoconazole $50 \mu \mathrm{g} ;(\mathrm{R})$ : résistant ; (S) : sensible,

Paramètres antifongiques (CMl et CMF) : Les CMl ont été déterminées pour les trois extraits de Capsicum (aqueux, éthanoliques et acétatique) (.tableau 3). Les souches les plus sensibles à ces extraits ont été retenues. Une activité antifongique significative des extraits éthanoliques et aqueux sur Alternaria sp.ont été observé. Ce pendant, l'extrait éthanolique s'est révélé le plus actif avec des valeurs de $\mathrm{CMl}$ allant de $84 \mathrm{mg} / \mathrm{mL}$ à $168 \mathrm{mg} / \mathrm{mL}$ conte $168 \mathrm{mg} / \mathrm{mL}$ à $336 \mathrm{mg} / \mathrm{mL}$. Ces mêmes extraits on eu des effets plus ou moins importante sur les souches Penicillium sp.et Fusarium sp., L'extrait acétatique a été moins actif sur l'ensemble des microbes.

Tableau 3 : Valeurs des paramètres antifongiques des différents extraits

\begin{tabular}{|c|c|c|c|c|c|c|c|c|c|}
\hline $\begin{array}{l}\text { Variétés } \\
\text { Capsicum }\end{array}$ & Extraits & Alternaria sp & & Penicillium sp & & Fusarium sp & & A.flavus & \\
\hline & & $\mathrm{CMI}$ & CMF & $\mathrm{CMI}$ & CMF & $\mathrm{CMI}$ & CMF & $\mathrm{CMI}$ & CMF \\
\hline Capsicum & Aqueux & 168 & 336 & 336 & 672 & 336 & 672 & - & - \\
\hline $\begin{array}{l}\text { Annuum } \\
\text { antillais }\end{array}$ & $\begin{array}{l}\text { Ethanolique } \\
\text { Acétatique }\end{array}$ & $\begin{array}{l}168 \\
168\end{array}$ & $\begin{array}{l}336 \\
672\end{array}$ & 168 & 336 & 336 & 672 & - & - \\
\hline Capsicum & Aqueux & 336 & 672 & - & - & - & ـ & - & - \\
\hline frutescens & Ethanolique & 168 & 336 & - & - & 336 & 672 & - & - \\
\hline soudanais & Acétatique & - & - & - & - & - & - & - & - \\
\hline Capsicum & Aqueux & 672 & 672 & - & - & - & - & - & - \\
\hline frutescens & Ethanolique & - & - & - & - & - & - & - & - \\
\hline attié & Acétatique & - & - & - & - & - & - & - & - \\
\hline Capsicum & Aqueux & 336 & 672 & - & - & - & - & - & - \\
\hline frutescens & Ethanolique & - & - & 336 & 672 & - & - & - & - \\
\hline doux & Acétatique & - & - & - & - & - & - & & - \\
\hline Capsicum & Aqueux & 336 & 672 & - & - & - & - & - & - \\
\hline frutescens & Ethanolique & 336 & 672 & - & - & 672 & 672 & - & - \\
\hline oiseau & Acétatique & - & - & - & - & - & - & 672 & 672 \\
\hline Capsicum & Aqueux & 168 & 168 & 168 & 672 & - & . & - & - \\
\hline chinense & Ethanoliqe & 84 & 84 & 168 & 336 & 336 & 672 & - & - \\
\hline pendulum & Acétatique & 168 & 336 & - & - & 336 & 672 & 336 & 168 \\
\hline
\end{tabular}




\section{DISCUSSION}

Cette étude a permis de réaliser des extraits aqueux, éthanolique et acétatique de six variétés de Capsicum et d'évaluer l'activité antifongique de ces extraits sur la croissance in vitro de cinq souches fongiques. Ces différentes variétés de Capsicum utilisées ont montré une activité antifongique sur des moisissures d'origine alimentaire. Ainsi, Capsicum chinense variété pendulum a été le plus actif sur Alternaria avec une zone d'inhibition de $19 \mathrm{~mm}$. Les variétés pendulum et antillais ont montré également une activité antifongique sur les souches Penicillium et Fusarium. Aucun extrait n'a pu inhiber la croissance de Aspergillus niger. Selon Wagner (1993) et Thangara et al. (2000), l'activité d'une substance végétale dépend de plusieurs facteurs dont le mode d'extraction et la concentration en principes actifs. Cichewicz et Thorpe (1996) ont montré que sur milieu gélosé ensemencé avec une souche cible, les tissus de Capsicum ont divers effets sur les microorganismes testés : une inhibition complète ou partielle ou une stimulation. Leurs travaux ont montré aussi que ces effets sont influencés pas la nature des tissus frais ou cuits. Les six variétés de Capsicum ont présentés des zones d'inhibitions pour les trois extraits sur les quatre souches fongiques. Les valeurs des zones d'inhibition vont de $16 \mathrm{~mm}$ à $18 \mathrm{~mm}$ pour Capsicum annuum; de $12 \mathrm{~mm}$ à $16 \mathrm{~mm}$ pour Capsicum frutescens et de $16 \mathrm{~mm}$ à $19 \mathrm{~mm}$ pour Capsicum chinenes. Ces résultats sont soutenues par ceux de Koffi-Nevry et al., (2012) qui ont montrés que Capsicum annuum et Capsicum frutescens présentaient des activités antibactériennes contre certaines bactéries pathogènes responsables de maladies d'origine alimentaire. Cependant, les diamètres d'inhibition induits par tous ces extraits restent la plupart inférieurs à ceux des antifongiques de références utilisés. Par ailleurs, la comparaison de l'effet antifongique des produits de références sur celui des extraits utilisés pourrait s'expliquer par le fait que les substances de référence sont des molécules pures, isolées et de concentration bien connue ce qui n'est pas forcement le cas avec les extraits éthanoliques et acétatiques qui sont des concentrés de constituant chimiques variés non purifié. Selon Biyitiet al., (2004), un extrait est considéré comme actif s'il enduit une zone d'inhibition supérieure ou égale à $10 \mathrm{~mm}$. Ainsi tous les trois extraits utilisés sont actifs

\section{CONCLUSION}

Les extraits aqueux, éthanoliques et acétatiques des variétés de piments (variétés antillais, attié, soudanais, doux, oiseau et chinense) possèdent une activité antifongique sur Alternaria sp., Fusarium sp., Penicillium sur Alternaria, Penicillium, Fusarium et Aspergillus flavus. Cependant, les extraits éthanoliques des variétés antillais et pendulum ont présentés une activité antifongique plus élevée que les extraits aqueux. D'autres auteurs ont montré aussi que les extraits éthanoliques d'une même espèce végétale sont plus actifs que les extraits aqueux (Traoré et al., 2012). De même, Zihiri et al. (2003) ont rapporté que l'extrait éthanolique de Mycroglossa pyrifolia est 100 fois plus actif que son extrait aqueux. Selon Thangara et al., (2000), il y a une différence de composition entre ces extraits et cette différence est liée au mode d'extraction utilisé. Cette observation est soutenue par les travaux de moroh et al. (2008) et de Bagré et al. (2011) qui ont montré que l'éthanol permet une meilleure concentration des principes actifs comparativement à l'extrait total aqueux. Selon DarouiMokaddem (2012), les valeurs des diamètres de la zone d'inhibition ont un rapport avec les valeurs de CMI recherchées c'est-à-dire que les extraits de Capsicum ayant des diamètres d'inhibition les plus élevés sont ceux qui possèderaient les CMI les plus faibles. Selon Bercher et al. (1991) lorsque le rapport CMF/CMI d'une substance est inférieur ou égal à quatre $(\leq 4)$, cette substance est jugée fongicide tandis qu'elle est dite fongistatique si ce rapport est supérieur à quatre (>4). Les trois extraits sont donc fongicides sur Alternaria sp, Penicillium sp, Fusarium sp et Aspergillus flavus selon les variétés. Les plus faibles valeurs des paramètres antifongiques ont été obtenues avec l'extrait éthanolique sur Alternaria. Pour ce microbe, la CMl était $84 \mathrm{mg} / \mathrm{mL}$. Les travaux de Kouassi (2012) ont montré que les variétés de Capsicum annuum et de Capsicum frutescens contiennent entre autre des alcaloïdes, des flavonoïdes, des tanins, des stéroïds et des polyphénols qui possèdent des propriétés antimicrobiennes. Les souches fongiques testées sont responsables d'affection épidermiques, des allergies respiratoires, des vomissements, des sinusites et produisent des toxines dans les aliments. L'activité antifongique des différents extraits des variétés de piments justifieraient l'utilisation du piment dans la médecine traditionnelle. L'ensemble de ces résultats constitue un argument scientifique important à l'usage de Capsicum dans la médecine traditionelle.

sp. et Aspergillus flavus. Capsicum chinense variété pendulum et Capsicum annuum variété antillais sont les variétés les plus actives sur les souches fongiques testées. Les extraits aqueux et éthanoliques ont présenté 
une activité antifongique significative cependant, La plus forte activité a été notée avec l'extrait éthanolique $70 \%$. Ce solvant concentrerait mieux les principes actifs de

\section{REFERENCES BIBLIOGRAPHIQUES}

Angeh JE, 2000. Isolation and characterization of antibacterial compounds present in members of Combretum section, Hypocrateroppsis. Ph D Thesis. University of Pretoria. Prétoria, Afrique du Sud. $210 \mathrm{pp}$.

Akinpelu DA, Aiyegoro OA, Okoh Al, 2008. In vitro antimicrobial and phytochemical properties of crude extract of stem bark of Afzelia Africana (Smith). African Journal of Biotechnology, 7 (20), 3665-3670.

Bagré I, Bahi C, Ouattara K, Zirihi GN, Djaman AJ, Coulibaly A, N'guessan JD, 2011. Étude botanique et exploration de l'activité antifongique de Morinda morindoides (Baker) Milne-Redh sur la croissance in vitro de Cryptococcus neoformans. Phytothérapie 9: 136-141.

Berche P, Gaillard JL, Simonet M, 1991. Les bactéries des infections humaines. Éditeur Flammarion, Médecine et Sciences, $660 \mathrm{p}$.

Biyiti LF, Meko'o DJL, Tamzc V, Amvam ZPH, 2004. Recherche de l'activité antibactérienne de quatre plantes médicinales camerounaises. Pharmacopée et Médecine Traditionnelle Africaine Vol.13, pp.11-20.

Biyiti LF, Tamze V, Nnanga N, Agbor AG, Gangouépieboji J, 2012. Formulation d'une pommade antibactérienne à base d'un extrait éthanolique des écorces du tronc de Tabernaemontana crassa Benth. Pharmacopée et Médecine Traditionnelle Africaine Vol.16.15 p.

Bouzouita N, Kachouri F, Ben HM, Chaabouni MM, 2008. Composition chimique et activités antioxydante, antimicrobienne et insecticide de l'huile essentielle de juniperus phoenicea. Journal de la Société Chimique de Tunisie 10, 119-125.

Cichewicz RH. et Thorpe PA, 1996. The antimicrobial properties of chilli peppers (Capsicum species) and their uses in Mayan medicine. Journal Ethnopharmologie. 52: 61-70.

Daroui-MH, 2012. Étude phytochimique et biologique des espèces Eucalyptus globulus (Myrtaceae, Smyrniumolusatrum (Apiaceae), Asteriscus maritimus et chysanthemus trifurcatum( Asterarceae). Thèse de Doctorat; Université Badji Mokhtar-Annaba, 198p.
Capsicum. Cette étude constitue un argument scientifique indéniable quant à l'utilisation traditionnelle des variétés de Capsicum dans le traitement de certaines infections.

Dorantes L, Colmenero R, Hermandez H, Mota L, Jaramillo ME, Fernandez E, Solanco C, 2000. Inhibition of growth of some foodborne pathogenic bacteria by Capsicum annuum extracts. International Journal of Food Microbiology, 57, 125-128.

Dosso M, Faye-Kette H, 2001. Savoir, Lire et Interpréter un Antibiogramme. A l'Attention du Bio Technologiste. INFAS/CHU de Treichville : Abidjan, $\mathrm{RCl}$.

El Koury $A, 2007$. Champignons Mycotoxinogènes et Ochratoxine A (OTA) et Aflatoxine B1(AFB1) dans les vignobles libanais: Occurrence et Origine. Thèse de Doctorat de l'Institut Nationnal de Polytechnique de Toulouse, France. 213p.

Elujoba AA, Odeleye OM. Ogunyemi CM, 2005. Traditionnal medicine development for medical and dental primary health care delivery system in Africa. African journal traditional complementary and Alternate Medecine, 2 :4661.

Hervert-Hernandez D, Sayago-Ayerdi SG, Goni I, 2010. Bioactive compounds of four hot pepper varieties (Capsicum annuum L.) : antioxidant capacity, and intestinal bioaccessibility. Journal of Agricultural and Food Chemistry, 58: 33993406.

Koffi-Nevry R, Kouassi C, Nanga Z, Koussémon M, Loukou G, 2012. Antibacterial activity of two bell pepper extracts: Capsicum annum L. and Capsicum frutenscens. International Journal of Food Properties, 15: 961-971, 2012.

Koné WM, Kamanzi AK, Terreaux C, Hostettmann K, Traore D, Dosso M, 2004. Traditional medicine in North Côte-d'Ivoire: screening of 50 medicinal plants for antibacterial activity. Journal of Ethnopharmacology, 93(1): 43-49.

Kra AKM, 2001. Évaluation et amélioration par séquençage chromatographique d'une action antifongique de MISCA contre Aspergillus fumigatus. Thèse. Biochimie UFR Biosciences. Université. Abidjan 126 pages.

Kouassi C, 2012. Potentialités bioactives et activité antimicrobienne des variétés de piment (Capsicum) cultivées en Côte d'Ivoire. Thèse de 
Doctorat ; Université d'Abobo-Adjamé, Abidjan, Côte d'lvoire, 161p.

Kouassi CK. et Koffi-Nevry, 2012. Évaluation de la connaissance et utilisation des variétés de piment (Capsicum) cultivées en Côte d' Ivoire. International Journal Biology Chemical Sciences (1): $175-785$.

Kporou KE, Kra AKM, Ouattara S, Guede-GuinaDjaman AJ, 2010. Amélioration par fractionnement chromatographique de l'activité anticandidosique d'un extrait hexanique de Mitracarpus scaber Zucc sur la croissance in vitro de Candida albicans et Candida tropicalis. Phytotheurapie, 8 : 290-294. :

Lee $\mathrm{KH},$. 2004. Current Developments in the Discovery and Design of New Drug Candidates from Plant Natural Product Leads. Journal of Natural products.,67, 273-283.

Moroh JLA, Bahi C, Djè K, Loukou YG, Guédé-Guina F, 2008. Étude de l'activité antibactérienne de l'extrait acétatique (EAC) de Morinda morindoides (Baker) milne-redheat (rubiaceae) sur la croissance in-vitro des souches d'Escherichia coli. Bull de la SR des Sciences de Liège, $77: 44-61$.

N'guessan JD, Coulibaly A, Ramanou A, Okou OC, Djaman AJ, Guédé-Guina F, 2007. Antibacterial activity of Thonningia Sanguinea against some multi-drug resistant strains of Salmonella enterica. African Health Sciences, 7 (3), 155158.

OMS, 2003. Médicaments essentiels et politiques pharmaceutiques: donner un soutien au pays pour produire le manque d'accès aux médicaments. Genève: OMS (rapport annuel 2002) $20 \mathrm{p}$.

Nobori T, Miurak K, Wu DJ, Takabayashik LA, Carson DA,1994. Detection of the cyclindependent kinase-4 inhibitor gene in multiple human cancers. Nature, 368 (6473), 753-756

Suffredini IB, Paciencia MLB, Varella AD, Younes RN, 2006. Antibacterial activity of brazilian amazon plant extracts. The Brazilian Journal Infectious Diseases., 10(6):400-2.

Tchiegang C,Maoundipa FP,Kapchie NV, 1999. Étude comparée de quelques constituants chimiques de deux types de piment (Capsicum annuum $L$.) pendant la conservation dans une saumure acide. Journal of Food Engineering, 42,117-123.

Thangara JHS, Adjei O, Allen B W, Portaels F, 2000. Invitro activity of ciprofloxacin, sparfloxacin, ofloxacin, amikacin and rifampicin against Ghanian isolates of Mycobacterium ulcerans. Journal Antimicrobial Agents Chemoter, 45 (2), 231-233.

Traoré Y, Ouattara K, Yéo D, Doumbia I, Coulibaly, 2012. Recherche des activités antifongique et antibactérienne des feuilles d'Annoma senegalens pers. (Annonnaceae). Journal of Applied biosciences $58: 4234-4242$.

Vinod KG, Amit R, Vikas KN, Kalishankar M, 2010. Antimicrobial activity of Spondias pinnata resin. Journal of Medicinal Plants Research. 4(16), pp. 1656-1661

Wagner H, 1993. Pharmazeutische Biologe. Drogen und irhe inhaltsstaffe, Gustav Fisher Verfag. Sturtgart-New-York, $50 \mathrm{p}$.

Zirihi G.et Kra AKM, 2003. Évaluation de l'activité antifongique de Microglossapyrifolia (Lamarck) O. Kuntze (Asteraceae) "Pymi » sur la croissance in vitro de Candida albicans. Rev Med PharmAfr 17: 11-18. 